\title{
ON K-SUPERQUADRATIC SET-VALUED FUNCTIONS
}

\author{
Katarzyna Troczka-Pawelec \\ Institute of Mathematics, Częstochowa Jan Dtugosz University \\ Czesstochowa, Poland \\ k.troczka@ajd.czest.pl
}

\begin{abstract}
In this paper we consider K-superquadratic set-valued functions. We will present here some connections between K-boundedness of K-superquadratic set-valued functions and K-semicontinuity of multifunctions of this kind.
\end{abstract}

Keywords: set-valued functions, quadratic set-valued functions, subquadratic real-valued functions

\section{Introduction}

Let $X=(X,+)$ be an arbitrary topological group. A real-valued function $f$ is called superquadratic if it fulfils inequality

$$
2 f(x)+2 f(y) \leq f(x+y)+f(x-y), \quad x, y \in X .
$$

If the sign $\leq$ in (1) is replaced by $\geq$, then $f$ is called subquadratic. The continuity problem of functions of this kind was considered in [1]. This problem was also considered in the class of set-valued functions. By the set-valued functions we understand functions of the type $F: X \rightarrow 2^{Y}$, where $X$ and $Y$ are given sets. Throughout this paper set-valued functions will be always denoted by capital letters.

A set-valued function $F$ is called subquadratic if it satisfies inclusion

$$
F(x+y)+F(x-y) \subset 2 F(x)+2 F(y), \quad x, y \in X
$$

and superquadratic, if it satisfies inclusion defined in this form

$$
2 F(x)+2 F(y) \subset F(x+y)+F(x-y), \quad x, y \in X .
$$

For single-valued real functions properties of subquadratic and superquadratic functions are quite analogous and, in view of the fact that if a function $f$ is subquadratic, then the function $-f$ is superquadratic and conversely, it is not 
necessary to investigate functions of these two kinds individually. In the case of set-valued functions the situation is different. Even if properties of subquadratic and superquadratic set-valued functions are similar, we have to prove them separately.

If the sign $\subset$ in the inclusions above is replaced by $=$, then $F$ is called quadratic set-valued function. The class of quadratic set-valued functions is an important subclass of the class of subquadratic and superquadratic set-valued functions. Quadratic set-valued functions have already extensive bibliography (see Smajdor [2], Henney [3] and Nikodem [4]).

The continuity problem of subquadratic and superquadratic set-valued functions was considered in [5] and [6].

Adding a cone $K$ in the space of values of a set-valued function $F$ lets us consider a K-superquadratic set-valued function, that is, solution of the inclusion

$$
F(x+y)+F(x-y) \subset 2 F(x)+2 F(y)+K, \quad x, y \in X
$$

where $F$ is defined on 2-divisible topological group $X$ with non-empty, compact and convex values in a locally convex topological vector space $Y$.

The concept of K-superquadraticity is related to real-valued superquadratic functions. Note, in the case when $F$ is a single-valued real function and $K=[0 ; \infty)$ we obtain the standard definition of superquadratic functionals (1).

If a set-valued function $F$ satisfies the following inclusion

$$
2 F(x)+2 F(y) \subset F(x+y)+F(x-y)+K, \quad x, y \in X
$$

then is called K-subquadratic. The K-continuity problem of multifunction of this kind was considered in [7] and [8]. It was proved that a K-subquadratic set-valued function, which is K-continuous at zero, $F(0)=\{0\}$ and locally K-bounded in $X$ is $\mathrm{K}$-continuous every where in $X$.

In this paper we will consider similar problem for K-superquadratic set-valued functions. Likewise as in functional analysis we can look for connections between $\mathrm{K}$-boundedness and K-semicontinuity of set-valued functions of this kind. Assuming $K=\{0\}$ in (4) and (5) we obtain the inclusions (2) and (3).

Let us start with the notations used in this paper. Let $Y$ be a topological vector space. We consider the family $n(Y)$ of all non-empty subsets of $Y$ as a topological space with the Hausdorff topology. In this topology the set

$$
N_{W}(A):=\{B \in n(Y): A \subset B+W, B \subset A+W\}
$$

where $W$ runs the base of neighbourhoods of zero in $Y$, form a base of neighbourhoods of a set $A \in n(Y)$. By $c c(Y)$ we denote the family of all compact and convex members of $n(Y)$. The term set-valued function will be abbreviated to the form s.v.f. 
Now we present here some definitions for the sake of completeness.

Recall that a set $K \subset Y$ is called a cone if $K+K \subset K$ and $s K \subset K$ for all $s \in(0 ; \infty)$.

\section{Definition 1.1}

A cone $K$ in a topological vector space $Y$ is said to be a normal cone if there exists a base $\Lambda$ of zero in $Y$ such that

$$
W=(W+K) \cap(W-K)
$$

for all $W \in \Lambda$.

\section{Definition 1.2}

An s.v.f. $F: X \rightarrow n(Y)$ is said to be $\mathrm{K}$-upper semi-continuous (abbreviated K-u.s.c.) at $x_{0} \in X$ if for every neighbourhood $V$ of zero in $Y$ there exists a neighbourhood $U$ of zero in $X$ such that

$$
F(x) \subset F\left(x_{0}\right)+V+K
$$

for every $x \in x_{0}+U$.

\section{Definition 1.3}

An s.v.f. $F: X \rightarrow n(Y)$ is said to be K-lower semi-continuous (abbreviated K-1.s.c.) at $x_{0} \in X$ if for every neighbourhood $V$ of zero in $Y$ there exists a neighbourhood $U$ of zero in $X$ such that

$$
F\left(x_{0}\right) \subset F(x)+V+K
$$

for every $x \in x_{0}+U$.

\section{Definition 1.4}

An s.v.f. $F: X \rightarrow n(Y)$ is said to be K-continuous at $x_{0} \in X$ if it is both $\mathrm{K}$-u.s.c. and K-l.s.c. at $x_{0} \in X$. It is said to be K-continuous if it is $\mathrm{K}$-continuous at each point of $X$.

Note that K-continuity of $F$ in the case where $K=\{0\}$ means its continuity with respect to the Hausdorff topology on $n(Y)$.

We will frequently use the following lemma.

\section{Lemma 1.1 ([7])}

Let $Y$ be a topological vector space and $K$ be a cone in $Y$. Let $A, B, C$ be nonempty subsets of $Y$ such that $A+C \subset B+C+K$. If $B$ is convex and $C$ is bounded then $A \subset \overline{B+K}$. 


\section{The main result}

In the proof of the main theorem, which will be presented here, we will often use four known lemmas (see Lemma 1.1, Lemma 1.3, Lemma 1.6 and Lemma 1.9 in [9]). The first lemma says that for a convex subset $A$ of an arbitrary real vector space $Y$ the equality $(s+t) A=s A+t A$ holds for every $s, t \geq 0$ (or $s, t \leq 0$ ). The second lemma says that in a real vector space $Y$ for two convex subsets $A, B$ the set $A+B$ is also convex. The next lemma says that if $A \subset Y$ is a closed set and $B \subset Y$ is a compact set, where $Y$ denotes a real topological vector space, then the set $A+B$ is closed. For any sets $A, B \subset Y$ where $Y$ denotes the same space as above, the inclusion $\bar{A}+\bar{B} \subset \overline{A+B}$ holds and equality holds if and only if the set $\bar{A}+\bar{B}$ is closed.

Note that for the cone $K$ the following remark holds.

\section{Remark 2.1}

Let $Y$ be a real topological vector space. If $K$ is a closed cone, then it is a cone with zero.

Let us adopt the following three definitions which are natural extension of the concept of the lower and upper boundedness for real-valued functions.

\section{Definition 2.1}

An s.v. function $F: X \rightarrow n(Y)$ is said to be $\mathrm{K}$-lower bounded on a set $A \subset X$ if there exists a bounded set $B \subset Y$ such that $F(x) \subset B+K$ for all $x \in A$.

\section{Definition 2.2}

An s.v. function $F: X \rightarrow n(Y)$ is said to be K-upper bounded on a set $A \subset X$ if there exists a bounded set $B \subset Y$ such that $F(x) \subset B-K$ for all $x \in A$.

\section{Definition 2.3}

An s.v. function $F: X \rightarrow n(Y)$ is said to be locally K-lower (upper) bounded in $X$ if for every $x \in X$ there exists a neighbourhood $U_{x}$ of zero in $X$ such that $F$ is $\mathrm{K}$-lower (upper) bounded on a set $x+U_{x}$. It is said to be locally K-bounded in $X$ if it is both locally K-lower and locally K-upper bounded in $X$.

\section{Theorem 2.1}

Let $X$ be a 2-divisible topological group, $Y$ locally convex topological real vector space and $K \subset Y$ a closed normal cone. If a $\mathrm{K}$-superquadratic s.v.f. $F: X \rightarrow c c(Y)$ is K-u.s.c. at zero, $F(0)=\{0\}$ and locally $\mathrm{K}$ - bounded in $X$ then it is $\mathrm{K}-1$. s.c. in $X$. 


\section{Proof:}

Suppose that $F$ is not K-l.s.c. at a point $z \in X$ i.e. there exists a neighbourhood $V$ of zero in $Y$ such that for every neighbourhood $U$ of zero in $X$, we can find $x_{u} \in X$ for which

$$
F(z) \nsubseteq F\left(z+x_{u}\right)+V+K
$$

Take a balanced convex neighbourhood $W$ of zero in $Y$ such that

$$
W \subset V
$$

and

$$
\overline{F\left(z+x_{u}\right)+W+K} \subset F\left(z+x_{u}\right)+V+K
$$

Then also

$$
F(z) \nsubseteq \overline{F\left(z+x_{u}\right)+W+K}
$$

We shall show by induction that

$$
F(z)+2^{s}\left(2^{s}-1\right) F\left(x_{u}\right) \nsubseteq \underline{F\left(z+2^{s} x_{u}\right)+2^{s} W+K}
$$

for every neighbourhood $U$ of zero in $X$ and $s \in N_{0}:=\{0,1,2,3 \ldots\}$. For $k=0$ condition (7) holds with respect to (6). We assume that (7) holds for $s=k$ and for every neighbourhood $U$ of zero in $X$. Let $s=k+1$. Putting $y=x$ in (4) and using condition $F(0)=\{0\}$ we have

$$
F(2 \mathrm{x}) \subset 4 F(x)+K .
$$

An easy induction shows

$$
F\left(2^{n} x\right) \subset 4^{n} F(x)+K,
$$

for $x \in X$ and for all positive integers $n$. By K-superquadraticity of $F$ and (8), we have

$$
\begin{gathered}
F\left(z+2^{k+1} x_{u}\right)+F(z)=F\left(z+2^{k} x_{u}+2^{k} x_{u}\right)+F\left(z+2^{k} x_{u}-2^{k} x_{u}\right) \subset \\
2 \mathrm{~F}\left(z+2^{k} x_{u}\right)+2 \mathrm{~F}\left(2^{k} x_{u}\right)+K \subset \\
2 \mathrm{~F}\left(z+2^{k} x_{u}\right)+2^{2 \mathrm{k}+1} F\left(x_{u}\right)+K .
\end{gathered}
$$

In view of the fact that for any sets $A, B \subset Y, \bar{A}+\bar{B} \subset \overline{A+B}$ we get

$$
\overline{F\left(z+2^{k} x_{u}\right)+2^{k} W+K}+K \subset \overline{F\left(z+2^{k} x_{u}\right)+2^{k} W+K}
$$


and consequently

$$
\overline{\overline{F\left(z+2^{k} x_{u}\right)+2^{k} W+K}+K} \subset \overline{F\left(z+2^{k} x_{u}\right)+2^{k} W+K} .
$$

By (7) and (10), we obtain

$$
F(z)+2^{k}\left(2^{k}-1\right) F\left(x_{u}\right) \nsubseteq \overline{\overline{F\left(z+2^{k} x_{u}\right)+2^{k} W+K}+K} .
$$

Notice that for a cone $K$ the equality $a K=K$ holds, for every $a \in(0 ; \infty)$. Hence

$$
2 F(z)+2^{k+1}\left(2^{k}-1\right) F\left(x_{u}\right) \nsubseteq \overline{\overline{2 F\left(z+2^{k} x_{u}\right)+2^{k+1} W+K}+K} .
$$

By (12) and Lemma 1.1

$$
\begin{aligned}
& 2 F(z)+2^{k+1}\left(2^{k}-1\right) F\left(x_{u}\right)+2^{2 \mathrm{k}+1} F\left(x_{u}\right) \nsubseteq \\
& 2 F\left(z+2^{k} x_{u}\right)+2^{k+1} W+K+2^{2 \mathrm{k}+1} F\left(x_{u}\right)+K
\end{aligned}
$$

In view of Remark $2.1 K$ is a cone with zero. Therefore, by above

$$
\begin{gathered}
2 F(z)+2^{k+1}\left(2^{k}-1\right) F\left(x_{u}\right)+2^{2 \mathrm{k}+1} F\left(x_{u}\right)+K \nsubseteq \\
2 F\left(z+2^{k} x_{u}\right)+2^{k+1} W+K+2^{2 \mathrm{k}+1} F\left(x_{u}\right)+K .
\end{gathered}
$$

In view of the fact that the sum of closed and compact sets is closed and for any sets $A, B \subset Y, \bar{A}+\bar{B}=\overline{A+B}$ in the case where $\bar{A}+\bar{B}$ is a closed set, we get

$$
\frac{\overline{2 F\left(z+2^{k} x_{u}\right)+2^{k+1} W+K}+2^{2 \mathrm{k}+1} F\left(x_{u}\right)}{2 F\left(z+2^{k} x_{u}\right)+2^{k+1} W+K+2^{2 \mathrm{k}+1} F\left(x_{u}\right)} .
$$

Since $K$ is a cone, by (9) we obtain

$$
\overline{\frac{2 F\left(z+2^{k} x_{u}\right)+2^{k+1} W+K+2^{2 \mathrm{k}+1} F\left(x_{u}\right)}{F\left(z+2^{k+1} x_{u}\right)+F(z)+2^{k+1} W+K} .}
$$

Since $F$ has closed values, we get

$$
\begin{gathered}
F(z)+\overline{F\left(z+2^{k+1} x_{u}\right)+2^{k+1} W+K}+K \subset \\
F\left(z+2^{k+1} x_{u}\right)+F(z)+2^{k+1} W+K \\
F
\end{gathered}
$$

Consequently, using (13)-(16), we conclude

$$
\begin{gathered}
2 F(z)+2^{k+1}\left(2^{k}-1\right) F\left(x_{u}\right)+2^{2 \mathrm{k}+1} F\left(x_{u}\right)+K \nsubseteq \\
F(z)+\frac{F\left(z+2^{k+1} x_{u}\right)+2^{k+1} W+K}{F}+K .
\end{gathered}
$$


By convexity of the sets $F\left(x_{u}\right), F(z)$ we obtain

$$
\begin{gathered}
F(z)+F(z)+2^{k+1}\left(2^{k+1}-1\right) F\left(x_{u}\right)+K \nsubseteq \\
F(z)+\frac{F\left(z+2^{k+1} x_{u}\right)+2^{k+1} W+K}{}+K .
\end{gathered}
$$

Therefore

$$
F(z)+2^{s}\left(2^{s}-1\right) F\left(x_{u}\right) \underline{\underline{F\left(z+2^{s} x_{u}\right)+2^{s} W+K}}
$$

for $s=k+1$, so that (7) is generally valid for all integers $s \geq 0$.

Since $K$ is a normal cone, there exists a base $\Lambda$ of neighbourhoods of zero in $Y$ such that $M=(M+K) \cap(M-K)$ for all $M \in \Lambda$. We can choose $W_{1} \in \Lambda$ and balanced neighbourhood $W_{2}$ of zero in $Y$ such that

$$
W_{2} \subset W_{1} \subset W
$$

Because $M \in \Lambda$. is $\mathrm{K}$-lower bounded on a neighbourhood of $z$, then there exists a neighbourhood $U_{0}$ of zero in $X$ and bounded set $B_{1} \subset Y$ such that

$$
F(z+t) \subset B_{1}+K, \quad t \in U_{0} .
$$

Since the set $B_{1}$ is bounded, there exists $\lambda_{1}>0$ such that

$$
B_{1} \subset \frac{1}{\lambda_{1}} W_{2} \text {. }
$$

Therefore, from the above

$$
F(z+t) \subset \frac{1}{\lambda_{1}} W_{2}+K, \quad t \in U_{0} .
$$

Because $F$ is K-upper bounded on a neighbourhood of $z$, then there exists a neighbourhood $U_{1}$ of zero in $X$ and bounded set $B_{2} \subset Y$ such that

$$
F(z+t) \subset B_{2}-K, \quad t \in U_{1}
$$

Since the set $B_{2}$ is bounded, there exists $\lambda_{2}>0$ such that

$$
B_{2} \subset \frac{1}{\lambda_{2}} W_{2} \text {. }
$$

Therefore, from the above

$$
F(z+t) \subset \frac{1}{\lambda_{2}} W_{2}-K, \quad t \in U_{1}
$$


Let $\lambda=\min \left\{\lambda_{1}, \lambda_{2}\right\}$. Since $W_{2}$ is balanced, we get

$$
F(z+t) \subset \frac{1}{\lambda} W_{2}+K \subset \frac{1}{\lambda} W_{1}+K, \quad t \in U_{0}
$$

and

$$
F(z+t) \subset \frac{1}{\lambda} W_{2}-K \subset \frac{1}{\lambda} W_{1}-K, \quad t \in U_{1} .
$$

By (17) and (18), we obtain

$$
F(z+t) \subset\left(\frac{1}{\lambda} W_{1}+K\right) \cap\left(\frac{1}{\lambda} W_{1}-K\right), \quad t \in U_{0} \cap U_{1} .
$$

Because the set $M \in \Lambda$, we have

$$
\left(\frac{1}{\lambda} W_{1}+K\right) \cap\left(\frac{1}{\lambda} W_{1}-K\right)=\frac{1}{\lambda} W_{1}
$$

and consequently the following inclusion holds

$$
F(z+t) \subset \frac{1}{\lambda} W_{1} \subset \frac{1}{\lambda} W,
$$

for every $t \in U_{0} \cap U_{1}$.

Let $k \in N$ be so large that

$$
2^{k}>\frac{3}{\lambda}
$$

Since $F$ is K-u.s.c. at zero and $F(0)=\{0\}$ there exists a neighbourhood $U_{2}$ of zero in $X$ such that

$$
U_{2} \subset \frac{1}{2^{k}}\left(U_{0} \cap U_{1}\right)
$$

and

$$
F(t) \subset \frac{1}{\lambda 2^{k}\left(2^{k}-1\right)} W+K, \quad t \in U_{2} .
$$

There exists $x_{u} \in U_{2}$ such that (7) holds.

By (22)

$$
2^{k} x_{u} \in\left(U_{0} \cap U_{1}\right)
$$


and by (23)

$$
F\left(x_{u}\right) \subset \frac{1}{\lambda 2^{k}\left(2^{k}-1\right)} W+K
$$

Let $a \in F\left(z+2^{k} x_{u}\right), b \in F(z), c \in F\left(x_{u}\right)$. By (20), (21), (24), (25), we obtain

$$
b+2^{k}\left(2^{k}-1\right) c-a \in \frac{1}{\lambda} W+\frac{1}{\lambda} W+K+\frac{1}{\lambda} W \subset 2^{k} W+K .
$$

Therefore

$$
b+2^{k}\left(2^{k}-1\right) c \in F\left(z+2^{k} x_{u}\right)+2^{k} W+K .
$$

We have proved that

$$
F(z)+2^{k}\left(2^{k}-1\right) F\left(x_{u}\right) \subset F\left(z+2^{k} x_{u}\right)+2^{k} W+K,
$$

which contradicts (7).

\section{Conclusions}

This article is the introduction to the discussion on the K-continuity problem for $\mathrm{K}$-superquadratic set-valued functions. In the theory of $\mathrm{K}$-subquadratic and K-superquadratic set-valued functions an important role is played by theorems giving possibly weak conditions under which such multifunctions are K-continuous. In this paper we have presented some connections between K-boundedness of K-superquadratic set-valued functions and K-semicontinuity of multifunctions of this kind.

\section{References}

[1] Kominek Z., Troczka-Pawelec K., Continuity of real valued subquadratic functions, Commentationes Mathematicae 2011, 51, 1, 71-75.

[2] Smajdor W., Subadditive and subquadratic set-valued functions, Prace Naukowe Uniwersytetu Śląskiego w Katowicach nr 889, Katowice 1987.

[3] Henney D., Quadratic set-valued functions, Ark. Mat. 1964, 2.

[4] Nikodem K., On quadratic set-valued functions, Publ. Math., Debrecen 1983, 30.

[5] Troczka-Pawelec K., Continuity of subquadratic set-valued functions, Demonstratio Mathematica 2012, XLV, 4, 939-946.

[6] Troczka-Pawelec K., Continuity of superquadratic set-valued functions, Scientific Issues Jan Długosz University in Częstochowa, Mathematics XVII, 2012.

[7] Troczka-Pawelec K., K-subquadratic set-valued functions, submitted.

[8] Troczka-Pawelec K., K-continuity of K-subquadratic set-valued functions, submitted.

[9] Nikodem K., K-convex and K-concave set-valued functions, Zeszyty Naukowe Politechniki Łódzkiej, nr 1989, 559. 